\title{
Boundary control problem for a dynamic Kirchhoff plate model with partial observations
}

\author{
Erik Hendrickson \\ Department of Computer Science and Mathematics \\ Arkansas State University, University, AR 72401, U.S.A. \\ and \\ Irena Lasiecka \\ Institute of Applied Mathematics \\ University of Virginia, Charlottesville, Virginia 22901, U.S.A.
}

\begin{abstract}
Boundary control problems of partially observed hyperbolic systems are considered. An algorithm based on FEM leading to a construction of a finite dimensional control is provided. In order to secure the stability of the algorithm, presence of boundary controls, yielding unbounded control operators in the semigroup model, necessitates introducing, prior to the FEM discretization, a specially designed regularization procedure where an additional boundary viscosity term is added. This stability and convergence of the overall regularized and discretized control problem is demonstrated. The theory is applied to a dynamic Kirchhoff plate with controls acting as the moments and boundary observations.
\end{abstract}

\section{Keywords}

Boundary control, Kirchhoff, compensator, regularization, stability

\section{ORIENTATION}

We consider the problem of the design of a boundary feedback control for a dynamic Kirchhoff plate model where only a partial observation of the state is available, for instance, a boundary observation. Since full knowledge of the state is not available, the primary problem centers around obtaining an estimate of the original state and using this estimate as if it were an exact measurement of the state to solve the original, deterministic control problem. In other words, a feedback control law, called the compensator, is generated using only information from an estimate of the state variable. The generalized linear-quadratic-Gaussian (LQG) theory supporting this method provides a state estimate that reconstructs the full state asymptotically in time and is optimal, in a suitable context, when Gaussian white noise processes pollute the input and output of the dynamical system. Hence, via the separation principle, the original problem is partitioned into solving a deterministic, linear quadratic optimal control problem and an optimal filtering (estimation) problem. The solutions to both the control and estimation problems 
are based on the solutions to associated Riccati equations with unbounded coefficients (as they arise in the context of boundary control).

Since the original system is infinite dimensional and practicality dictates that both the estimator and compensator must be finite dimensional. A natural way to circumvent this problem is to construct a finite dimensional approximation (eg. via finite elements) of the original control system that will uniformly (in the parameter of discretization) retain the control theoretic properties of the resulting closed loop system, such as the desired performance level and asymptotic stability. To accomplish this, the stability and convergence of solutions to finite dimensional Riccati equations must be provided. The final goal will be to show that the finite dimensional compensator, which is based on a finite dimensional approximation to the estimate and solutions to finite dimensional Riccati equations and when applied to the original system, will produce near optimal performance of the closed loop system. Moreover, the uniform stability of the resulting system should be preserved.

Problems related to finite dimensional approximations to infinite dimensional compensators with boundary controls have received considerable attention in the literature (see Curtain (1986), Ito (1990), Lasiecka (1995), Schumacher (1983) and references therein). However, all these works provide satisfactory convergence and uniform stabilizability results for classes of systems satisfying the so-called spectrum determined growth condition. These, in turn, are essentially restricted to delay and parabolic-like dynamics. Moreover, in these cases, stability analysis is much simplified as it amounts to the analysis of the spectrum. The situation is much more complicated in the case of hyperbolic dynamics, where the location of the spectrum does not necessarily determine the stability. In fact, approximations of hyperbolic compensator systems were analyzed in Lasiecka (1992). The results in Lasiecka (1992) provide the optimal theory for bounded control operators, but in the case of unbounded control actions (as in this paper), they require a certain trace-type condition. Indeed, a discrete analogue of (5) is to be satisfied uniformly in the parameter of discretization. As it turns out, this condition may fail for certain popular approximation techniques such as FEM in 2-d problems (Hendrickson, 1993a, 1995). The goal of the present paper is to remove the above restriction. Thus, the novel features of the present paper, with respect to prior literature, are:

(i) hyperbolicity of the dynamics;

(ii) unboundedness of the control actions, such as those which arise in boundary/point control problems;

(iii) no need to impose the discrete trace condition (see Lasiecka, 1992).

In what follows, we shall first treat an abstract hyperbolic system and then show how the abstract theory yields the results for a model of a Kirchhoff plate.

\section{FORMULATION OF THE PROBLEM}

Let $H_{0}$ and $U$ be Hilbert spaces. Let $A_{0}: H_{0} \supset \mathcal{D}\left(A_{0}\right) \rightarrow H_{0}$ be a positive, self-adjoint operator on $H_{0}$, and $B_{0}: U \rightarrow\left[\mathcal{D}\left(A_{0}^{1 / 2}\right)\right]^{\prime}$ be a control operator (i.e. $A_{0}^{-1 / 2} B_{0} \in \mathcal{L}\left(U, H_{0}\right)$ ). 
Notice that $B_{0}$ is unbounded: $U \rightarrow H_{0}$. We consider the following second order dynamics,

$$
\begin{aligned}
& z_{t t}(t)+A_{0} z(t)+D z_{t}(t)=B_{0} u(t) \quad \text { on } \quad\left[\mathcal{D}\left(A_{0}^{1 / 2}\right)\right]^{\prime} \\
& z(t=0)=z_{0} \in \mathcal{D}\left(A_{0}^{1 / 2}\right), \quad z_{t}(t=0)=z_{1} \in H_{0}
\end{aligned}
$$

Here, the operator $D$ is assumed nonnegative, self-adjoint and bounded on $H_{0}$. It is well known that (1) can be rewritten as the first order equation

$$
\begin{aligned}
& x_{t}(t)=A x(t)+B u(t) \quad \text { on } \quad[\mathcal{D}(A)]^{\prime}, \\
& x(t=0)=x_{0}=\left(z_{0}, z_{1}\right) \in H \equiv \mathcal{D}\left(A_{0}^{1 / 2}\right) \times H_{0}, \text { where } \\
& A \equiv\left[\begin{array}{cc}
0 & I \\
-A_{0} & -D
\end{array}\right] ; \quad B \equiv\left[\begin{array}{c}
0 \\
B_{0}
\end{array}\right] ; \quad x \equiv\left(z, z_{t}\right)^{T}
\end{aligned}
$$

and $A$ is a generator of a $C_{0}$-semigroup $e^{A t}$ defined on the Hilbert space $H$. With the dynamics described by (2) we associate a partial observation given by

$y(t)=C x(t)$

where $C \in \mathcal{L}(H: Y), \mathrm{Y}$ is a given Hilbert space representing the output space. We shall be considering the class of problems with the control operator $B$, generally unbounded, satisfying the following trace assumption,

$\int_{0}^{T}\left\|B^{*} e^{A^{*} t} x\right\|_{U}^{2} d t \leq C_{T}\|x\|_{H}^{2}, x \in \mathcal{D}\left(A^{*}\right)$

where $(B u, v)_{H}=\left(u, B^{*} v\right)_{U} u \in U, v \in \mathcal{D}\left(B^{*}\right) \supset \mathcal{D}\left(A^{*}\right)$.

Remark $B$ : continuous from $U \rightarrow\left[\mathcal{D}\left(\mathcal{A}^{*}\right)\right]^{\prime}$ implies that $A^{-1} B \in \mathcal{L}(\mathcal{U}, \mathcal{H})$. It is well known (see Lasiecka and Triggiani, 1991a) that assumption (5) is satisfied for a large class of hyperbolic models including boundary control models for waves and plates.

The associated control problem is to develop a feedback control law, based only on the observed state, which minimizes (over $u \in L_{2}(0, \infty ; U)$ ) the following cost functional

$J(u, x(u))=\int_{0}^{\infty}\|R x\|_{H}^{2}+\|u\|_{U}^{2} d t, \quad R \in \mathcal{L}(H)$.

Following ideas developed in finite dimensional estimator theory, we propose the following structure for the estimator. The dynamical estimator for the system (2), (4) is given by

$$
\begin{aligned}
& w_{t}(t)=A w(t)+B u(t)+K(y(t)-C w(t)) \text { on }(\mathcal{D}(A))^{\prime} \\
& w(t=0)=w_{0} \in H
\end{aligned}
$$

where the operator $K \in \mathcal{L}(Y ; H)$. 
Remark One can show that, by virtue of hypothesis (5), for a given control $u \in$ $L_{2}(0, T ; U)$, the solution $(\mathrm{x}, \mathrm{w})$ of $(2),(7)$ is contained in $C([0, T] ; H \times H)$ and is continuous with respect to the data, (see Lasiecka and Triggiani, 1991a).

Assuming that the operator $K^{*}$ is stabilizing for the pair $\left(A^{*}, C^{*}\right)$ on $H$, one can prove (see Lasiecka, 1992) that the estimator is uniformly exponentially stable, i.e.,

$\|x(t)-w(t)\|_{H} \leq C e^{-\omega t}\left\|x_{0}-w_{0}\right\|_{H}$, for some $C, \omega>0$.

Thus, the estimator $w(t)$ reconstructs the dynamics of $x(t)$, asymptotically in $t$. Since the full state $x(t)$ is not available and any reasonable feedback law must be based on the available information, we consider feedbacks of the form,

$u(t)=F w(t)$, where $F: H \rightarrow U$, closed and densely defined

\section{OPTIMAL F-D COMPENSATOR AND CLOSED LOOP SYSTEM}

Following the LQG theory, the optimal state feedback control law F and Kalman filter gain $\mathrm{K}$ are to be sought in the form:

$F=-B^{*} P, \quad K=\hat{P} C^{*}$,

where $P, \hat{P} \in \mathcal{L}(H)$ are the positive, semidefinite solutions of the following algebraic Riccati equations (ARE),

$$
\begin{aligned}
& \left(A^{*} P x, y\right)_{H}+(P A x, y)_{H}+\left(R^{*} R x, y\right)_{H}=\left(B^{*} P x, B^{*} P y\right)_{U}, x, y \in \mathcal{D}(A), \\
& (A \hat{P} x, y)_{H}+\left(\hat{P} A^{*} x, y\right)_{H}+\left(Q^{*} Q x, y\right)_{H}=(C \hat{P} x, C \hat{P} y)_{Y}, x, y \in \mathcal{D}\left(A^{*}\right)
\end{aligned}
$$

and $Q \in \mathcal{L}(H)$.

It is well known that the conditions associated with the unique solvability of the AREs (11), (12) are, together with (5),

$$
(A, B),\left(A^{*}, C^{*}\right),(A, Q), \text { and }\left(A^{*}, R^{*}\right) \quad \text { are stabilizable on } \mathrm{H} \text {. }
$$

In fact, the above result is standard if $B$ is bounded, see Balakrishnan (1981). In the case considered here, the situation is more complicated due to the unboundedness of $B$. However, due to the trace assumption (5), a recent theory of Flandoli, Lasiecka and Triggiani (1988) applies and provides, subject to (13), an existence and uniqueness of a solution $P \in \mathcal{L}(H)$ to (11). Moreover, it is proven that the gain operator is properly defined on the domain of the generator, i.e. $B^{*} P \in \mathcal{L}(\mathcal{D}(A) ; H)$. Hence the feedback law, in (9), is unbounded but densely defined on $\mathrm{H}$ and the nonlinear term in (11) is well-defined on a dense set. If in addition the following smoothing assumption on $\mathrm{R}$ is satisfied

$R^{*} R A \in \mathcal{L}(H)$ 
then it is shown in Da Prato, Lasiecka and Triggiani (1986) that $B^{*} P \in \mathcal{L}(H ; U)$, implying that the gain, $B^{*} P$, is a bounded operator. Using the above described result on the solvability of Riccati Equations, (11), (12), it was shown in Lasiecka (1992) that under the conditions (5), (13), the optimal compensator, described by the system

$\left[\begin{array}{c}x \\ w\end{array}\right]_{t}=\mathcal{A}\left[\begin{array}{c}x \\ w\end{array}\right], \quad$ where $\mathcal{A}=\left[\begin{array}{cc}A & B F \\ K C & A+B F-K C\end{array}\right]$,

with $\mathrm{F}$ and $\mathrm{K}$ given by (10) is exponentially stable, i.e.

$\left\|e^{\mathcal{A t}}\right\|_{\mathcal{L}(H \times H)} \leq C e^{-\omega t}$

for some $\omega>0$.

We seek to design a finite dimensional control, $u_{h}(y)=u_{h}(C x)$, where $h \rightarrow 0$ (the parameter of discretization), such that the original system (2), with $u_{h}$, will provide near optimal performance, i.e. $J\left(u_{h}, x\left(u_{h}\right)\right) \rightarrow J\left(u^{0}, x\left(u^{0}\right)\right)$, as $h \rightarrow 0$ and $u^{0}$ corresponds to the optimal, infinite dimensional control of the original system. In addition, we also aim to preserve the stability of the resulting closed-loop system.

The precise formulation of the approximation algorithm and of the main abstract results are given in the following sections.

\section{REGULARIZATION}

Our first step is to regularize the original continuous problem. To accomplish this, we introduce the operator,

$A_{\epsilon} \equiv A-\epsilon B B^{*}$,

where $\epsilon>0$ is a parameter of regularization tending to zero. It was shown in Hendrickson and Lasiecka (1993b) that $A_{\epsilon}$ generates a $C_{0}$-semigroup such that

$\left\|e^{A_{\epsilon} t}\right\|_{\mathcal{L}(H)} \leq M e^{\omega_{0} t}, \quad \omega_{0}>0$

uniformly in $\epsilon>0$. The following results are also supported in Hendrickson and Lasiecka (1993b):

$\left(A_{\epsilon}, B\right),\left(A_{\epsilon}^{*}, C^{*}\right) \quad$ are uniformly $($ in $\epsilon>0)$ stabilizable, and
$\left(A_{\epsilon}^{*}, Q\right),\left(A_{\epsilon}, R\right) \quad$ are uniformly $($ in $\epsilon>0)$ detectable.

Note that these results do not follow from standard perturbation theory, since $\epsilon B B^{*}$ is not a bounded perturbation, and are necessary to assert the unique solvability of the algebraic Riccati Equations (11), (12) with $A$ replaced by $A_{\epsilon}$. 


\section{APPROXIMATION}

We shall approximate the regularized problem. To accomplish this we introduce the following approximation subspaces and operators. Let $h$ denote the discretization parameter which is assumed to tend to zero. $V_{h} \subset \mathcal{D}\left(A_{0}^{1 / 2}\right)$ be a finite dimensional subspace. Let $\pi_{h}$ represent the orthogonal projection of $H_{0}$ onto $V_{h}$ with the properties:

$\exists y_{h}=\pi_{h} y \in V_{h} \ni\left\|\pi_{h} y-y\right\|_{H_{0}} \rightarrow 0, \quad y \in H_{0}$,

$\left\|\pi_{h} y-y\right\|_{\mathcal{D}\left(A_{0}^{1 / 2}\right)} \rightarrow 0, \quad y \in \mathcal{D}\left(A_{0}^{1 / 2}\right)$.

Assumptions on $A_{0}$ : Let $A_{0 h}: V_{h} \rightarrow V_{h}$ be a Galerkin approximation of $A$ such that

$\left(A_{0 h} x_{h}, \xi_{h}\right)_{H_{0}}=\left(A_{0} x_{h}, \xi_{h}\right)_{H_{0}}, \quad x_{h}, \xi_{h} \in V_{h} . \quad$ Hence

$\left\|\left(A_{0}^{-1}-A_{0 h}{ }^{-1} \pi_{h}\right) x\right\|_{\mathcal{D}\left(A_{0}^{1 / 2}\right)} \rightarrow 0, \quad x \in H_{0}$.

Assumptions on $D$ : Let $D_{h}: V_{h} \rightarrow V_{h}$ be a Galerkin approximation of $D$ such that

$\left(D_{h} x_{h}, \xi_{h}\right)_{H_{0}}=\left(D x_{h}, \xi_{h}\right)_{H_{0}}, \quad x_{h}, \xi_{h} \in V_{h}$.

Assumptions on $B_{0}$ : Let $B_{0 h}: U \rightarrow V_{h}$ be such that

$\left\|B_{0 h}^{*} \pi_{h}-B_{0}^{*}\right\|_{\mathcal{D}\left(A_{0}^{1 / 2}\right) \rightarrow U} \rightarrow 0$

here

$\left(B_{0}^{*} v, g\right)_{U}=\left(v, B_{0} g\right)_{H_{0}} \quad$ for $\quad g \in U, v \in \mathcal{D}\left(B_{0}^{*}\right) \supset \mathcal{D}\left(A_{0}{ }^{1 / 2}\right)$,

$\left\|A_{0}^{-1}\left(B_{0 h}-B_{0}\right)\right\|_{U \rightarrow \mathcal{D}\left(A_{0}^{1 / 2}\right)} \rightarrow 0$

$\left\|\left(A_{0 h}{ }^{-1}-A_{0}^{-1}\right) B_{0 h}\right\|_{U \rightarrow \mathcal{D}\left(A_{0}{ }^{1 / 2}\right)} \rightarrow 0$.

Define $\bar{V}_{h} \equiv V_{h} \times V_{h}$ and $\bar{\pi}_{h}$ is the orthogonal projection of $H$ onto $\bar{V}_{h}$ with

$\overline{\pi_{h}}=\left[\begin{array}{cc}\pi_{h} & 0 \\ 0 & \pi_{h}\end{array}\right]$.

Also, $A_{h}: \bar{V}_{h} \rightarrow \bar{V}_{h}$ and $B_{h}: U \rightarrow \bar{V}_{h}$, where

$A_{h} \equiv\left[\begin{array}{cc}0 & \pi_{h} \\ -A_{0 h} & -D_{h}\end{array}\right], \quad B_{h} \equiv\left[\begin{array}{c}0 \\ B_{0 h}\end{array}\right], \quad A_{h, \epsilon} \equiv A_{h}-\epsilon B_{h}^{*} B_{h}$.

Notice that $B_{h}^{*} v_{h}=B_{0 h}^{*} v_{2 h}$, where $v_{h}=\left(v_{1 h}, v_{2 h}\right) \subset \bar{V}_{h}$, and that the above approximation properties on $A_{0}, B_{0}, D$ are the usual consistency and stability properties satisfied by most approximation schemes including standard finite element (FE) and finite difference (FD) methods.

The finite dimensional gains associated with (10) are

$F_{h, \epsilon}=B_{h}^{*} P_{h, \epsilon}, \quad K_{h, \epsilon}=\hat{P_{h, \epsilon}} \pi_{h} C^{*}$, 
where $P_{h, c}, \hat{P_{h, c}} \in \mathcal{L}\left(\bar{V}_{h}\right)$ are positive, self-adjoint, finite dimensional operators which solve the following Riccati equations for all $x_{h}, y_{h} \in \bar{V}_{h}$,

$$
\begin{aligned}
& \left(A_{h, \epsilon}{ }^{*} P_{h, \epsilon} x_{h}, y_{h}\right)_{H}+\left(P_{h, \epsilon} A_{h, \epsilon} x_{h}, y_{h}\right)_{H}+\left(R_{h}^{*} R_{h} x_{h}, y_{h}\right)_{H}=\left(B_{h}^{*} P_{h, \epsilon} x_{h}, B_{h}^{*} P_{h, \epsilon} y_{h}\right)_{U}, \\
& \left(A_{h, \epsilon} \hat{P_{h, \epsilon}} x_{h}, y_{h}\right)_{H}+\left(\hat{P_{h, \epsilon}} A_{h, \epsilon}{ }^{*} x_{h}, y_{h}\right)_{H}+\left(Q_{h}^{*} Q_{h} x_{h}, y_{h}\right)_{H}=\left(C \hat{P_{h, \epsilon}} x_{h}, C \hat{P_{h, \epsilon}} y_{h}\right)_{Y} .
\end{aligned}
$$

In the above equations, $R_{h}, Q_{h}$ are suitable, convergent approximations of $R, Q$, i.e. $R_{h} \rightarrow R, Q_{h} \rightarrow Q$ strongly in $H$.

The regularized, finite dimensional control, $u_{h}(t)=F_{h, \epsilon} w_{h}(t)$, when applied to the original system gives rise to the following compensator problem generated by the operator, $\mathcal{A}_{h, \varepsilon}: \mathcal{H} \rightarrow \mathcal{H}$, where $\mathcal{H} \equiv H \times H$,

$\mathcal{A}_{h, \epsilon}=\left(\begin{array}{ll}A & B F_{h, \epsilon} \pi_{h} \\ K_{h, \epsilon} \pi_{h} C & A_{h, \epsilon} \pi_{h}+B_{h} F_{h, \epsilon} \pi_{h}-K_{h, \epsilon} \pi_{h} C\end{array}\right)$.

\section{ABSTRACT RESULT}

The main goal is to show that $e^{\mathcal{A}_{h, \epsilon} t}$ converges as $h, \epsilon \rightarrow 0$ to the original compensator design described by $e^{\mathcal{A} t}$, where $\mathcal{A}$ is given in (15). This is given in the theorem below, whose proof is given in Hendrickson and Lasiecka (1995).

Theorem 1 We assume continuous hypotheses (5), (13), (14) and approximation hypotheses (21)-(28). In addition, we shall assume that either $Q$ or $C$ is compact and that

$(D x, x)_{H_{0}} \geq d\|x\|_{H_{0}}^{2}, \quad d>0$,

$R_{h}$ satisfies a discrete counterpart to (14), i.e.

$\left\|R_{h}^{*} R_{h} A_{h}\right\|_{\mathcal{L}(H)} \leq C$, uniformly in $h>0$.

Then, for all $\bar{x}=(x, w) \in \mathcal{H} \equiv H \times H$,

$\lim _{\epsilon \rightarrow 0} \lim _{h \rightarrow 0} \sup _{t \geq 0}\left\|e^{\mathcal{A}_{h, \epsilon} t} \bar{x}-e^{\mathcal{A} t} \bar{x}\right\|_{\mathcal{H}}=0$.

(Convergence of controls)

$\lim _{\epsilon \rightarrow 0} \lim _{h \rightarrow 0}\left\|u_{h, \epsilon}-u\right\|_{L_{2}(0 \infty ; U)}=0$,

(Convergence of Performance Index)

$\lim _{\epsilon \rightarrow 0} \lim _{h \rightarrow 0} J\left(u_{h, \epsilon}, x\left(u_{h, \epsilon}\right)\right)-J(u, x(u))=0$,

(Uniform stability)

There exists $\omega_{0}>0$ such that for all $\bar{x}=(x, 0)$,

$\left\|e^{\mathcal{A}_{h, \epsilon} t} \bar{x}\right\|_{\mathcal{H}} \leq C e^{-\omega_{0} t}\|\bar{x}\|_{\mathcal{H}}$. 
Remark Under the minimal approximation hypotheses, i.e. the standard stability and consistency hypotheses, the main result of the Main Theorem provides an algorithm for the construction of a finite dimensional control $u_{h, \epsilon}(t)$ which, when inserted into the original system, gives a near optimal performance of the system. The resulting compensator system retains the uniform stability properties (see (39)), provided that the initial condition for the estimator $(\mathrm{w})$ equation, i.e. $w_{0}$ is sufficiently regular. Typically $w_{0}=0$, so that this regularity requirement is satisfied automatically. But, the necessity of assuming higher regularity for $w_{0}$ results from an interesting new feature of the problem which is the lack of a uniform $C_{0}$-semigroup estimate for the operator $e^{\mathcal{A}_{h, \epsilon} t}$. We show that $e^{\mathcal{A}_{h, \epsilon} t}$ is, in fact, a one time integrated semigroup (Arendt (1983)) while $\left\|e^{\mathcal{A}_{h, \epsilon} t}\right\|_{\mathcal{L}(\mathcal{H})}$ is of order $\left(\frac{1}{\sqrt{\epsilon}}\right)$. The main goal of this paper is to show how the abstract theory applies to a model of the Kirchhoff plate.

\section{KIRCHHOFF PLATE WITH BOUNDARY CONTROL AND BOUNDARY OBSERVATION}

The following Kirchhoff plate model motivates our abstract theory. We shall consider the equation of the Kirchhoff plate with boundary control $u(t)$ acting as a bending moment and the state variable $z(t)$ representing the vertical displacement of the plate.

$$
\begin{array}{ll}
k z_{t t}-\gamma \Delta z_{t t}+\Delta^{2} z+d\left(z_{t}-\gamma \Delta z_{t}\right)=0 & \text { on } \Omega \times(0, \infty) \\
z(x, t)=0, \quad \Delta z(x, t)=u(t) & \text { on } \Gamma \times(0, \infty) \equiv \Sigma, \\
z(x, 0)=z_{0}(x), \quad z_{t}(x, 0)=z_{1}(x) & \text { on } \Omega,
\end{array}
$$

where $\Omega$ is an open domain in $R^{2}$ with boundary $\Gamma$ of sufficient regularity. k pertains to the flexibility of the plate. $\gamma>0$ is a parameter that is proportional to the square of the plate thickness. The term $\gamma \Delta z_{t t}$ represents rotational forces (which are neglected in some models). $d \geq 0$ is the damping coefficient. $u(t)$ acts as a boundary control in the form of a bending moment. Notice that the uncontrolled and undamped $(u(t)=0, d=0)$ system is unstable, i.e. there are infinitely many eigenvalues on the imaginary axis. The natural energy associated with the model is

$E(t)=\int_{\Omega}|\Delta z(t)|^{2}+\gamma\left|\nabla z_{t}\right|^{2}+\left|z_{t}\right|^{2} d \Omega$.

With model (40) we associate the boundary observation given by

$C\left(\begin{array}{l}z \\ z_{t}\end{array}\right)=\left.\frac{\partial}{\partial \nu} z(x, t)\right|_{\Gamma}$

The cost functional is defined as

$J(u, z(u))=\int_{0}^{\infty}\left[\int_{\Gamma} u^{2}(x, t) d \Gamma+\int_{\Omega}\left|\nabla\left(z(x, t)-z_{d}(x)\right)\right|^{2} d \Omega\right] d t$,

where $z_{d}(x) \in H^{2}(\Omega)$ is a given element. Physically, $z_{d}(x)$ represents a desired trajectory that we wish to track. Hence we seek to minimize the energy of the control and the 
gradient of the error between the actual trajectory and the desired trajectory. Since only a partial (boundary) observation is available, we need to determine the state estimator and then the desired infinite dimensional control will be of the form

$u^{0}(t)=F\left(w(t), w_{t}(t)\right)$

where $w, w_{t}$ is an estimator for (40). In order to construct the estimator according to the theory, we rewrite the partial differential equation as an abstract equation within the framework of section 1 .

To put problem (40) into an abstract framework, we introduce the following spaces and operators.

$U=L_{2}(\Gamma) ; \quad H_{0}=H_{0}^{1}(\Omega) \quad$ with the inner product

$$
(u, v)_{H_{0}}=\int_{\Omega} u v d \Omega+\gamma \int_{\Omega} \nabla u \nabla v d \Omega
$$

$$
\begin{aligned}
A_{D} u \equiv-\Delta u & : u \in \mathcal{D}\left(A_{D}\right) \equiv H^{2}(\Omega) \cap H_{0}^{1}(\Omega) \\
A_{D} & : L_{2}(\Omega) \supset \mathcal{D}\left(A_{D}\right) \rightarrow L_{2}(\Omega), \\
A_{0} & : H^{2}(\Omega) \cap H_{0}^{1}(\Omega) \rightarrow L_{2}(\Omega), \text { where } A_{0}=\left(I+\gamma A_{D}\right)^{-1} A_{D}^{2}
\end{aligned}
$$

$\mathcal{B}_{0} g=\left(I+\gamma A_{D}\right)^{-1} A_{D} D_{0} g$, where $D_{0}$ is the Dirichlet map defined by $D_{0}: L_{2}(\Gamma) \rightarrow L_{2}(\Omega)$ and $D_{0} g=v$ implies $\left\{\Delta v=0\right.$ on $\left.\Omega ;\left.v\right|_{\Gamma}=g\right\}$.

$\left(\mathcal{B}_{0} g, h\right)_{H_{0}}=\left(A_{D} D_{0} g, h\right)_{\Omega}=\left(g, D_{0}^{*} A_{D} h\right)_{\Gamma}=\left(g, \frac{\partial}{\partial \nu} h\right)_{\Gamma}$.

Using (44) and (45) we verify that

$$
\begin{aligned}
\|u\|_{\mathcal{D}\left(A_{0}^{1 / 2}\right)}^{2} & =\left(A_{0} u, u\right)_{H_{0}}=\left(\left(I+\gamma A_{D}\right)\left(I+\gamma A_{D}\right)^{-1} A_{D}^{2} u, u\right)_{L_{2}(\Omega)} \\
& =\left\|A_{D} u\right\|_{L_{2}(\Omega)}^{2}=\|\Delta u\|_{L_{2}(\Omega)}^{2} .
\end{aligned}
$$

Hence $E(t)=\|z(t)\|_{\mathcal{D}\left(A_{0}^{1 / 2}\right)}^{2}+\left\|z_{t}(t)\right\|_{H_{0}}^{2}$. With the above notation, (40) is equivalent to the abstract model (1), i.e.,

$z_{t t}+\left(I+\gamma A_{D}\right)^{-1} A_{D}^{2} z+d z_{t}=\left(I+\gamma A_{D}\right)^{-1} A_{D} D_{0} u$ on $\left[\mathcal{D}\left(A_{D}\right)\right]^{\prime}$

It is shown (see Lasiecka and Triggiani, 1991a) that

(i) A, defined by (3), is a generator of a $C_{0}$-semigroup on $H \equiv \mathcal{D}\left(A_{0}^{1 / 2}\right) \times H_{0}(\Omega)$,

(ii) Hypothesis (5), which amounts to the trace regularity (Lasiecka and Triggiani, $1991 \mathrm{ab})$.

$$
\int_{0}^{T} \int_{\Gamma}\left|\frac{\partial}{\partial \nu} \Delta z(t)\right|^{2} d x d t \leq C_{T}\left[\|z(0)\|_{H^{2}(\Omega)}^{2}+\left\|z_{t}(0)\right\|_{H^{1}(\Omega)}^{2}\right]
$$


which is shown to be satisfied for all $z(t), z_{t}(t)$ solutions to (40) with $u=0$. Notice that (47) does not follow a priori from the regularity of the solution (see standard trace theory in Kesavan (1989)), rather it is an independent trace regularity result.

(iii) The original problem with pair $(A, B)$ is exponentially stabilizable with the boundary feedback $u(t)$. It has been shown (Horn and Lasiecka (1994), Lasiecka and Triggiani (1991ab)) that the above open loop problem is uniformly stabilizable in the space $H^{2}(\Omega) \times H^{1}(\Omega)$ by means of a suitable boundary feedback control, $u(t)=F\left(z_{t}(t)\right) \in L_{2}\left(0 \infty ; L_{2}(\Gamma)\right)$. (For more stability and controllability results on the Kirchhoff plate with other boundary conditions, see Horn and Lasiecka (1994), Lagnese and Liones (1989), Lasiecka and Triggiani (1991ab) and references therein.) In fact, it was shown in Horn and Lasiecka (1994) that with a feedback control of the form,

$$
u(t)=-\frac{\partial}{\partial \nu} z_{t} \in L_{2}\left(0 \infty ; L_{2}(\Gamma)\right)
$$

the energy, $E(t)$, of the system given by (41) decays exponentially.

(iv) The observation $C$ given by (42) is a bounded operator, $C: H \rightarrow Y \equiv L_{2}(\Gamma)$, by virtue of the Trace Theorem (Pazy (1986)), and is, in fact, compact.

(v) The operator $R$ associated with (43) (WLOG assume $z_{d}=0$ ) has form

$$
R\left(\begin{array}{l}
z \\
z_{t}
\end{array}\right)=\left(\begin{array}{l}
A_{D}^{-1 / 2} z \\
0
\end{array}\right),
$$

which is clearly in $\mathcal{L}(H)$ and moreover, since

$$
R^{*} R A\left(\begin{array}{l}
z \\
z_{t}
\end{array}\right)=\left(\begin{array}{l}
A_{D}^{-1} z_{t} \\
0
\end{array}\right)
$$

(14) is satisfied. Indeed, from (49)

$$
\begin{aligned}
& \left\|R^{*} R A\left(\begin{array}{c}
z \\
z_{t}
\end{array}\right)\right\|_{H}=\left\|A_{D}^{-1} z_{t}\right\|_{\mathcal{D}\left(A_{0}^{1 / 2}\right)}=\left\|\left(I+\gamma A_{D}\right)^{1 / 2}\left(I+\gamma A_{D}\right)^{-1 / 2} A_{D} A_{D}^{-1} z_{t}\right\|_{L_{2}(\Omega)} \\
& \leq C\left\|z_{t}\right\|_{L_{2}(\Omega)} \leq C\left\|\left(\begin{array}{c}
z \\
z_{t}
\end{array}\right)\right\|_{H} .
\end{aligned}
$$

(vi) If $d>0$, then the operator $D$ is coercive, i.e.,

$$
(D x, x)_{H_{0}}=d \int_{\Omega}\left(I+\gamma A_{D}\right)\left(I+\gamma A_{D}\right)^{-1} A_{D} x \cdot x d \Omega
$$

and using Green's formula and Poincare Inequality

$$
=d \int_{\Omega}|\nabla x|^{2} d \Omega \geq d_{1}\|x\|_{H_{0}^{1}(\Omega)}^{2}=d_{1}\|x\|_{H_{0}}^{2} .
$$


One can show by the Liapunov function technique (see Horn and Lasiecka, 1994) that the uncontrolled system is exponentially stable, hence for any $Q \in \mathcal{L}(H)$, the pairs $\left(A^{*}, Q\right)$, $(A, R)$ are detectable and $\left(A^{*}, C^{*}\right)$ is stabilizable.

Therefore, all the assumptions imposed on the continuous model are satisfied and the infinite dimensional estimator takes the form

$$
\begin{aligned}
& w_{t t}-\gamma \Delta w_{t t}+\Delta^{2} w+d \Delta w_{t}=K\left(\left.\frac{\partial}{\partial \nu} z_{t}\right|_{\Gamma}-\left.\frac{\partial}{\partial \nu} w_{t}\right|_{\Gamma}\right), \\
& w=0, \quad \Delta w=u \quad \text { on } \Sigma, \quad w(t=0)=w_{t}(t=0)=0 . \\
& K \in \mathcal{L}\left(L_{2}(\Gamma) \rightarrow H^{2}(\Omega) \times H_{0}^{1}(\Omega)\right) \text { is given by } \\
& K g=\hat{P} C^{*} g=\hat{P}\left[\begin{array}{l}
A_{D}^{-1} D g \\
0
\end{array}\right] .
\end{aligned}
$$

It can easily be computed that $C^{*} g=\left[\begin{array}{l}A_{D}^{-1} \\ 0\end{array}\right]$ and $C^{*}$ is bounded (and compact): $L_{2}(\Gamma) \rightarrow$ $H$. The operator $\hat{P}$ is the unique, positive definite solution to a Riccati equation (12) with an arbitrary, bounded $Q$. The control $u(t)$ is given in feedback form (see Hendrickson and Lasiecka, 1993b) as

$$
u(t)=-\frac{\partial}{\partial \nu} P_{2}\left(w, w_{t}\right)
$$

where $P=\left[P_{1}, P_{2}\right]$ satisfies the Riccati equation (11). Theory in (Da Prato, Lasiecka and Triggiani (1986)) together with (49) gives

$$
\frac{\partial}{\partial \nu} P_{2} \in \mathcal{L}\left(H^{2}(\Omega) \times H_{0}^{1}(\Omega) \rightarrow L_{2}(\Gamma)\right)
$$

Hence, the gain operator $F$ is bounded, despite the unboundedness of $B$.

Since our main task is to construct a finite dimensional control which converges to the original one and is based on a finite dimensional estimator, we introduce approximating spaces and approximating operators and show that all the hypotheses of the Theorem 1 are satisfied.

Let $V_{h} \subset H^{2}(\Omega) \cap H_{0}^{1}(\Omega)$ be a space of Hermite cubics with orthogonal projection $\pi_{h}: H^{2}(\Omega) \cap H_{0}^{1}(\Omega) \rightarrow V_{h}$. We define $A_{h 0}: \quad V_{h} \rightarrow V_{h}$ by the formula

$$
\left(A_{0 h} x_{h}, \xi_{h}\right)_{H_{0}}=\int_{\Omega}\left(I+\gamma A_{D}\right) A_{0 h} u_{h} v_{h} d \Omega \equiv\left(A_{0} x_{h}, \xi_{h}\right)_{H_{0}}=\int_{\Omega} \Delta u_{h} \Delta v_{h} d \Omega
$$

where (54) results from the self-adjointness of $A_{D}$ and taking into account zero boundary conditions for $u_{h}$ and $v_{h}$ on $\Gamma$. We also introduce the boundary operator, $B_{0 h} g=\pi_{h} B_{0} g$, defined via duality by

$$
\begin{aligned}
& \left(g, B_{0 h}^{*} u_{h}\right)_{U}=\left(B_{0 h} g, u_{h}\right)_{H_{0}}=\left(B_{0} g, u_{h}\right)_{H_{0}}, \quad \text { and } \\
& \left(B_{h}\left(\begin{array}{c}
x_{1 h} \\
x_{2 h}
\end{array}\right),\left(\begin{array}{l}
y_{1 h} \\
y_{2 h}
\end{array}\right)\right)_{H}=\left(B_{0 h} x_{2 h}, y_{2 h}\right)_{H_{0}}
\end{aligned}
$$




$$
\begin{aligned}
& =\left(\left(I+\gamma A_{D}\right)\left(I+\gamma A_{D}\right)^{-1} A_{D} D_{0} x_{2 h}, y_{2 h}\right)_{L_{2}(\Omega)} \\
& =\left(x_{2 h}, \frac{\partial}{\partial \nu} y_{2 h}\right)_{L_{2}(\Gamma)} .
\end{aligned}
$$

It has been verified in (Hendrickson (1993a)) that standard approximation hypotheses (21)-(28) are satisfied by the above operators. As an approximation of operator $R^{*} R$ we simply take its projection onto $\bar{V}_{h}$ with respect to the $H$ norm. This way we have

$\left\|R_{h}\left(\begin{array}{c}z_{h} \\ z_{t h}\end{array}\right)\right\|_{H}^{2}=\left\|R\left(\begin{array}{c}z_{h} \\ z_{t h}\end{array}\right)\right\|_{H}^{2}=\left\|\nabla z_{h}\right\|_{L_{2}(\Omega)}$.

It can also be shown that $R_{h}^{*} R_{h}$ satisfies the discrete assumption (35). Moreover, it is straightforward to show (see Hendrickson and Lasiecka, 1993b) that all the approximating assumptions (21)-(28) are satisfied.

Therefore, all the assumptions of Theorem 1 are satisfied and we are in a position to apply its conclusions. We can now construct a finite dimensional compensator that is based only on the available information through the partial observation.

Numerical results illustrating this theory are given in Hendrickson (1995).

\section{REFERENCES}

Arendt, W. (1983) Vector Valued Laplace Transforms and Cauchy Problems. Israel Journal of Mathematical, 59, 327-352.

Balakrishnan A.V. (1981). Applied Functional Analysis. Springer Verlag.

Curtain, R.F. and Salamon, D. (1986) Finite dimensional compensators for infinite dimensional systems with unbounded input operators. SIAM Journal of Optimization 24, 797-816.

Da Prato, G., Lasiecka, I. and Triggiani, R. (1986) A direct study of riccati equations arising in hyperbolic boundary control problems. Journal of Differential Equations, 26-47.

Flandoli, F., Lasiecka, I. and Triggiani, R. (1988) Algebraic riccati equations with nonsmoothing observations arising in hyperbolic and Euler-Bernoulli boundary control problems. Annali di Matematica Pura et. Applicata 153, 307-382.

Hendrickson, E. (1993a) Approximation and regularization methods for the riccati operator of the undamped Kirchhoff plate. Master's thesis, University of Virginia.

Hendrickson, E. (1995) Compensator design for the Kirchhoff plate model with boundary control. Journal of Applied Mathematics and Computer Science 5, no. 1.

Hendrickson, E. and Lasiecka, I. (1993b) Numerical approximations and regularizations of Riccati equations arising in hyperbolic dynamics with unbounded control operators. Computational Optimization and Applications 2, 343-390.

Hendrickson, E. and Lasiecka, I. (1995) Finite dimensional approximations of boundary control problems arising in partially observed hyperbolic systems. Dynamics of Continuous, Discrete and Impulsive Systems (to appear).

Horn, M.A. and Lasiecka, I. (1994) Asymptotic behavior with respect to thickness of boundary stabilizing feedback for the Kirchhoff plate. Journal of Differential Equations 114, no. 2, 396-433. 
Kazufumi, I. (1990) Finite dimensional compensators for infinite dimensional systems via Galerkin-type approximations. SIAM Journal of Control and Optimization 28, 12511269.

Kesavan, S. (1989) Topics in Functional Analysis. John Wiley and Sons.

Lagnese, J.E. and Liones, J.L. (1989) Modelling Analysis and Control of thin Plates. Masson.

Lasiecka, I. (1992) Galerkin approximations of infinite dimensional compensators for flexible structures with unbounded control action. Acta Applicandae Mathematicae 28, 101-133.

Lasiecka, I. (1995) Finite element approximations of compensator design for analytic generators with fully unbounded controls and observations. SIAM Journal of Control and Optimization 33, 67-88.

Lasiecka, I. and Triggiani, R. (1991a) Differential and algebraic riccati equations with applications to boundary and point control problems: Continuous and approximation theory. Springer Verlag.

Lasiecka, I. and Triggiani, R. (1991b) Exact controllability and uniform stabilization of Kirchhoff plates with boundary control only on $\left.\Delta w\right|_{\Sigma}$ and homogeneous boundary displacement. Journal of Differential Equations 93, 62-101.

Pazy, A. (1986) Semigroups of linear operators and applications to partial differential equations. Springer Verlag.

Schumacher, J.M. (1983) A direct approach to compensator design for distributed parameter systems. SIAM Journal of Control and Optimization 21, 823-836.

Triggiani, R. (1975) On the stabilizability problem in Banach space. Journal of Mathematical Analysis and Applications 54, 383-403. 